\title{
Pulmonary infection in Wegener granulomatosis and idiopathic pulmonary fibrosis
}

\author{
A G Richter, ${ }^{1}$ R A Stockley, ${ }^{1}$ L Harper, ${ }^{2}$ D R Thickett ${ }^{1}$
}

\begin{abstract}
- Additional methods are published online only at http:// thorax.bmj.com/content/vol64/ issue8

${ }^{1}$ Lung Injury and Fibrosis Treatment Programme and Lung Immunobiology Group, School of Clinical and Experimental Medicine, College of Medical and Dental Sciences, University of Birmingham, Birmingham, UK:

${ }^{2}$ Department of Renal Immunobiology, School of Immunity and Infection, College of Medical and Dental Sciences, University of Birmingham,

Birmingham, UK
\end{abstract}

Correspondence to:

Dr D Thickett, Lung Investigation

Unit, 1st floor Nuffield House,

Birmingham B15 2TH, UK;

d.thickett@bham.ac.uk

Received 30 October 2008 Accepted 12 March 2009

Published online first 8 April 2009

\section{ABSTRACT}

Rationale: Wegener granulomatosis (WG) has previously been associated with increased nasal carriage of Staphylococcus aureus, but no studies have investigated the occurrence of pathogen growth in the lower airways. objectives: To culture bronchoalveolar lavage fluid (BALF) from patients with WG, patients with idiopathic pulmonary fibrosis (IPF) and normal controls.

Methods: 33 patients with WG, 22 with IPF and 8 normal controls underwent bronchoscopy and bronchoalveolar lavage. Quantitative culture established bacterial levels in the lower airways. Culture experiments were designed to investigate whether BALF is a supportive environment for $S$ aureus growth. BALF cytokines were measured by ELISA.

Results: Pathogens were commonly grown from BALF of patients with WG and those with IPF. $S$ aureus was particularly associated with patients with WG both in relapse and in remission. BALF levels of interleukin 1 receptor antagonist (IL1ra) were statistically significantly elevated in those patients who grew a pathogen from lavage fluid. BALF from patients with WG and IPF stimulated $S$ aureus growth compared with normal lavage fluid.

Conclusions: Pathogens are more commonly isolated from BALF from patients with WG than from that of patients with IPF or normal controls, and with a different culture profile. IL1ra was associated with pathogen growth in WG and IPF. WG BALF is a trophic environment for $S$ aureus growth. Pulmonologists treating patients with acute or relapsing WG should consider bronchoscopic microbiological sampling and consider antibiotics with antistaphylococcal activity.

Wegener granulomatosis (WG) is a small vessel systemic vasculitis characterised by antineutrophil cytoplasmic antibodies (ANCAs) directed against proteinase-3 (PR-3). A role for infection in WG was first postulated by Frederick Wegener in 1936, but a more substantial argument was put forward in 1980, when a retrospective trial suggested that nearly $50 \%$ of relapses were provoked by infection. ${ }^{1}$ Nasal carriage of Staphylococcus aureus is increased in WG, and is associated with a higher relapse rate. ${ }^{2}$ How this relates to relapse and whether a similar process affects the lower respiratory tract is unknown.

It is unclear why patients with WG have increased nasal carriage of $S$ aureus. A controlled cytokine response is essential for bacterial clearance and resolution of inflammation. However, experiments in acute respiratory distress syndrome have shown that elevated interleukin 6 (IL6), IL1 receptor antagonist (IL1ra) and IL1 $\beta$ levels in bronchoalveolar lavage fluid (BALF) are associated with a promotive growth environment for $S$ aureus. ${ }^{3}{ }^{4}$ These studies suggest that a proinflammatory environment may be permissive for $S$ aureus growth, raising the possibility of a cause and effect relationship. Several reports have suggested the importance of cytokines in the inflammatory response seen in WG, but the clinical implications of these measurements have not been fully established.

Idiopathic pulmonary fibrosis (IPF) is a chronic interstitial lung disease associated with damage to the alveolar capillary barrier. A number of viruses have been implicated in IPF pathogenesis, ${ }^{5-7}$ but studies have not established whether bacteria in the lower airways are also present or play a proinflammatory role in patients with IPF. The current study was designed to establish the prevalence of lower airway pathogen growth in patients with WG or IPF and in normal controls, and then to determine any relationship with clinical or radiological findings. We went on to establish BALF levels of IL6, IL1ra and IL1 $\beta$ cytokines and lastly investigated whether WG BALF promotes $S$ aureus growth in vitro.

\section{METHODS}

\section{Patient recruitment}

Thirty-three patients with WG meeting the classification criteria according to the Chapel Hill consensus statement were recruited. ${ }^{8}$ Patients were enrolled consecutively as they presented at University Hospital Birmingham from 2003 to 2008 with new or relapsing disease, and remission patients were consecutively recruited from clinic at routine appointment. Disease onset, relapse and remission definitions were defined according to European League against Rheumatism recommendations. ${ }^{9}$ Disease activity in WG was monitored using the Birmingham Vasculitis Activity Score (BVAS). ${ }^{10}$

Contraindications to enrolment for patients with WG were refractory hypoxia (arterial oxygen tension $\left(\mathrm{PaO}_{2}\right) /$ fractional inspired oxyen $\left(\mathrm{FiO}_{2}\right)$ ratio ( $\mathrm{P}$ :F ratio) $<300$ on $40 \%$ oxygen), the need for ventilatory support (continuous positive airway pressure (CPAP), non-invase ventilation (NIV) or intermittent positive pressure ventilation (IPPV)) or continuous renal replacement therapy.

\section{Idiopathic pulmonary fibrosis}

We used IPF as a disease control which has not been associated with $S$ aureus nasal carriage and as a comparator group who also receive immunosuppression in order to enable comment about the disease-specific nature of lower airway $S$ aureus infection and for the influence of immunosuppres- 
sion to be addressed. Twenty-two patients diagnosed with IPF according to current American Thoracic Society (ATS) criteria ${ }^{11}$ were recruited sequentially at first presentation to our interstitial lung disease clinic before immunosuppressive treatment.

No patients with IPF who had a history or clinical features to suggest active pulmonary infection within the last 4 weeks prior to bronchoscopy were included. Eight normal volunteers free from respiratory disease were recruited as controls. The study was conducted according to the Declaration of Helsinki. All patients gave informed consent and this study was approved by the local ethics committee (South Birmingham, UK, LREC ref 2003/166).

\section{Imaging}

Patients with WG and those with IPF underwent high resolution CT (HRCT) scanning. Scans were read blinded to conditions by a respiratory specialist (DRT) and a radiologist (PG), and assessed for features previously noted in a review of pulmonary findings in WG. ${ }^{12}$

\section{Pulmonary function testing}

Forced vital capacity (FVC) was measured using the Jaeger Compact system (Viasys Healthcare, Basingstoke, UK). Total lung diffusing capacity for carbon monoxide (TLCO) was measured by a single-breath technique (Jaeger Compact system; Viasys Healthcare). Results are expressed as the percentage of predicted values.

\section{Bronchoalveolar lavage (BAL)}

All patients underwent BAL according to national guidelines. ${ }^{13}$ To prevent nasal contamination, patients were intubated through the mouth. A nasal swab was performed for culture to look for matched nasal and lower airway bacteriology.

\section{Repeat bronchoscopy}

All patients with WG were invited to undergo a repeat bronchoscopy to establish whether there was evidence of persistent colonisation-7 patients with WG agreed to have repeat bronchoscopy entirely for research purposes after induction of remission; 4 others agreed to repeat BAL as they needed bronchoscopy for the assessment of disease relapse. Nine patients with IPF agreed to repeat bronchoscopy after at least 3 months treatment with prednisolone, azathioprine and latterly $N$-acetylcysteine.

\section{Cytokine measurements}

BALF cytokine levels were measured by ELISA (R\&D Systems, Systems, UK) according to the manufacturer's instructions.

\section{Quantitative culture}

Serial 10-fold dilutions $\left(10^{4}-10^{8}\right)$ of the original BALF specimen were made in normal saline. An inoculum of each dilution was plated on chocolate and blood agar. Bacterial numbers at 24 and $48 \mathrm{~h}$ were estimated by two investigators, blinded to conditions, and expressed as colony-forming units (CFU)/ml BALF.

\section{Culture experiments}

To assess whether BALF is a permissive environment for $S$ aureus growth, a laboratory $S$ aureus (National Collection of Type Cultures, 6571, Oxford, UK) was incubated with BALF from 10 patients with WG, 10 patients with IPF and 6 normal controls. The $S$ aureus was cultured in brain heart infusion (BHI). An inoculum of $10^{-5}$ bacteria per $\mathrm{ml}$, determined using a haematocytometer, was added to $1 \mathrm{ml}$ of BALF or $0.9 \%$ saline as a control in a 50:50 mix with $1 \mathrm{ml}$ of BHI. Following an overnight incubation, 1:10 dilutions of the broth were made and dilutions of $10^{-4}-10^{-7}$ were inoculated onto chocolate agar plates, in triplicate. The plates were read, after $24 \mathrm{~h}$ incubation at $37^{\circ} \mathrm{C}$ and $5 \% \mathrm{CO}_{2}$, by two investigators blinded to the variable conditions. Preliminary experiments revealed that at the dilutions performed, the initial protein content did not effect $S$ aureus growth (data not shown). BALF experiments were repeated following heat inactivation of the proteins in the BALF for $10 \mathrm{~min}$ at $80^{\circ} \mathrm{C}$.

\section{Statistics}

Non-parametric data were assessed by Kolmogorov-Smirnoff test and are presented as median and interquartile range (IOR). Differences in pathogen growth patterns between cohorts and also between groups of patients with different WG activity were examined using Fisher exact test (FET). Cytokine analysis was performed using a Kruskal-Wallis test followed by a Dunn test to look at differences between cohorts and then look at differences within a disease group when a pathogen was present. Culture experiments had a Kruskal-Wallis test applied, and Dunn test was used to compare groups. A p value of $\leqslant 0.05$ was considered statistically significant. Statistics were performed using SPSS 15.

\section{RESULTS}

\section{Demographics}

At presentation, patients with WG were classified as acute (first ever presentation), relapse or remission based on their BVAS and clinical presentation. Clinical features of the individual activity groups, the 22 patients with IPF and 8 normal controls are described in table 1 . Three of $11(27 \%)$ acute patients underwent bronchoscopy before immunosuppression with steroids and cyclophosphamide, and 2/10 (20\%) remission patients were on no medication ( $60 \%$ steroids, $30 \%$ azathioprine, $20 \%$ cyclophosphamide and 10\% methotrexate). All relapse patients were on immunosuppressive medication at the time of bronchoscopy (83\% steroids, 25\% cyclophosphamide, 33\% azathioprine, $16 \%$ each mycophenolate, rituximab and infliximab). Patients receiving cyclophosphamide had co-trimoxazole chemoprophylaxis. Pathogen growth was unrelated to individual immunosuppressive therapies in active or remission patients (data not shown). Of the 16 patients who were on co-trimoxazole chemoprophylaxis, 10 grew a pathogen of which 6 were $S$ aureus. No patients with IPF had received treatment prior to the first bronchoscopy.

\section{Microbiology of first bronchoscopy after enrolment}

Pathogens were grown from 64\% (21/33) of WG BALF compared with $36 \%(8 / 22)$ of IPF (FET, $p=0.058)$ and $0 \%(0 /$ 8 ) of normal controls (FET, $p=0.001$ ). $S$ aureus grew in the BALF of $40 \%(13 / 33)$ of all patients with WG compared with $0 \%$ $(0 / 22)$ of patients with IPF (FET, $p=0.001)$. Other bacteria were identified equally in the two disease groups. No organisms were grown in the BALF from normal control subjects (table 2).

\section{BALF pathogen growth in the different WG activity groups}

Pathogens were more commonly grown in WG disease relapse $(83 \%(10 / 12))$ and remission $(70 \%(7 / 10))$ than at first acute presentation $(36 \%(4 / 11))$ although this did not reach statistical significance (FET, $\mathrm{p}=0.076$ ). $S$ aureus was statistically more likely to be grown in the relapse $(50 \%(6 / 12))$ and remission 
Table 1 Clinical features of the patient groups at the time of first bronchoscopy

\begin{tabular}{llllll}
\hline & Acute & Relapse & Remission & Normal & IPF \\
\hline Patient numbers & 11 & 12 & 10 & 8 & 22 \\
Sex: male & 6 & 7 & 5 & 3 & 16 \\
Age (mean) & 52 & 59 & 50 & 45 & 70 \\
Smoking: never/ex/current & $5 / 6 / 0$ & $4 / 7 / 1$ & $3 / 7 / 0$ & $2 / 5 / 1$ & $5 / 12 / 3$ \\
Pack years (mean) & 19 & 19 & 21 & 13 & 30 \\
FVC (mean, SE) & $108(3.7)$ & $109(4.3)$ & $112(7.2)$ & $\mathrm{N} / \mathrm{A}$ & $73(6.6)$ \\
TLCO (mean, SE) & $90(4.7)$ & $87(4.6)$ & $88(4.7)$ & $\mathrm{N} / \mathrm{A}$ & $49(3.4)$ \\
CRP (mean, SE) & $23(6.0)$ & $45(26.0)$ & $5.8(5.3)$ & $<1$ & $6(10.3)$ \\
ANCA positivity & $100 \%$ & $71 \%$ & $33 \%$ & $0 \%$ & $0 \%$ \\
PR3 positivity & $73 \%$ & $50 \%$ & $17 \%$ & $0 \%$ & $0 \%$ \\
MPO positivity & $18 \%$ & $14 \%$ & $0 \%$ & $0 \%$ & $0 \%$ \\
BVAS (mean, SE) & $5.4(1.4)$ & $5.6(1.6)$ & $0.5(0.3)$ & $0 \%$ & $0 \%$ \\
VDI (mean, SE) & $3.2(0.7)$ & $4.3(0.6)$ & $2(0.4)$ & $0 \%$ & $0 \%$ \\
Bronchiectasis & $18 \%$ & $43 \%$ & $50 \%$ & $\mathrm{~N} / \mathrm{A}$ & $74 \% *$ \\
Fibrosis & $73 \%$ & $36 \%$ & $33 \%$ & $\mathrm{~N} / \mathrm{A}$ & $100 \%$ \\
Cavities & $18 \%$ & $50 \%$ & $17 \%$ & $\mathrm{~N} / \mathrm{A}$ & 0 \\
Nodules & $91 \%$ & $71 \%$ & $66 \%$ & $\mathrm{~N} / \mathrm{A}$ & $32 \%$ \\
Emphysema & $18 \%$ & $18 \%$ & $22 \%$ & $\mathrm{~N} / \mathrm{A}$ & $32 \%$ \\
Haemoptysis & $9 \%$ & $14 \%$ & $0 \%$ & $\mathrm{~N} / \mathrm{A}$ & $0 \%$ \\
\hline
\end{tabular}

Characteristics relate to the time of patients' first bronchoscopy. Data are presented as the mean (SE). Normal C-reactive protein (CRP) at our institution is $<10$.

In Wegener granulomatosis, pulmonary fibrosis was limited focal disease in all but one case.

ANCA, antineutrophil cytoplasmic antibody; BVAS, Birmingham Vasculitis Activity Score; FVC, forced vital capacity (\% predicted);

IPF, idiopathic pulmonary fibrosis; MPO, myeloperoxidase antibody (serum); N/A, not applicable; PR-3, proteinase-3 antibody

(serum); TLCO, total lung diffusing capacity for carbon monoxide (\% predicted); VDI, vasculitis damage index.

${ }^{*}$ Bronchiectasis in patients with IPF describes traction bronchiectasis.

group $(60 \%(6 / 10))$ than in the first acute presentation with WG $(8.3 \%(1 / 12))$ (FET, $p=0.031)$.

Five of 13 (38\%) patients with WG who grew $S$ aureus in the BALF had only mixed normal flora grown from their nasal swab. Two of $12(17 \%)$ patients who grew $S$ aureus from their nasal swab had a sterile BALF. The remaining samples were concordant, and all $S$ aureus were methicillin sensitive.

\section{Sequential BAL microbiology}

There were no differences in the bacterial growth patterns from the first or second WG BALF. Three of 11 sequential patients with WG grew pathogens in both their BALF cultures, although the bacteria grown were different, giving no evidence of colonisation. Three patients grew a pathogen in the first culture but not in the second. Four patients grew a pathogen only in the second culture. A pathogen was more commonly grown on the second bronchoscopy although this was not statistically significant (FET, $\mathrm{p}=0.076$ ).
Five of nine patients with IPF who underwent sequential bronchoscopy had two sequential sterile cultures. Two patients grew pathogens only on the first occasion and two grew pathogens only on the repeat BAL. There was no increased likelihood of growing a pathogen on the second BAL culture $(p=0.376)$.

Pathogen growth related to clinical-radiological findings in WG Most patients with WG (91\%) had radiographic pulmonary abnormalities (table 1), with cavities more likely to occur in the disease relapse group $(p=0.031)$. The presence of a pathogen in WG BALF was not associated with bronchiectasis (FET, $p=0.761$ ), cavities $(p=0.399)$, fibrosis $(p=0.724)$ or nodules $(p=0.555)$. There were no statistically significant differences in blood Creactive protein (CRP) or white cell count in patients with WG and IPF who had evidence of bacterial infection (data not shown).

BALF cytokine levels and the relationship with pathogen growth BALF levels of IL1 $\alpha$ (Dunn test, $p=0.048)$, IL1 $\beta(p=0.003)$, IL1ra $(p<0.0001)$ and IL6 $(p=0.008)$ were elevated in patients

Table 2 Frequency of pathogens occurring in WG, IPF and normal controls

\begin{tabular}{|c|c|c|c|c|}
\hline \multirow[b]{2}{*}{ Organism } & \multicolumn{2}{|l|}{ WG* } & \multicolumn{2}{|l|}{ IPF } \\
\hline & No. of BALs & $\mathrm{CFU} / \mathrm{ml}$ & No. of BALs & $\mathrm{CFU} / \mathrm{ml}$ \\
\hline Staphylococcus aureus & 13 & $3 \times 10^{5}$ & 0 & 0 \\
\hline Haemophilus influenzae & 3 & $5 \times 10^{6}$ & 2 & $6 \times 10^{5}$ \\
\hline Haemophilus parainfluenzae & 3 & $2 \times 10^{4}$ & 2 & $1 \times 10^{5}$ \\
\hline Streptococcus pneumoniae & 1 & $5 \times 10^{7}$ & 1 & $1 \times 10^{5}$ \\
\hline Moraxella catarrhalis & 2 & $1 \times 10^{5}$ & 1 & $5 \times 10^{5}$ \\
\hline Pseudomonas aeruginosa & 1 & $1 \times 10^{6}$ & 1 & $4 \times 10^{5}$ \\
\hline Proteus mirabilis & 0 & 0 & 1 & $4 \times 10^{4}$ \\
\hline Klebsiella pneumoniae & 1 & $4 \times 10^{5}$ & 0 & 0 \\
\hline
\end{tabular}

Bacterial growth is presented as mean colony-forming units (CFU)/ml. No normal controls grew pathogenic bacteria in their bronchoalveolar lavage fluid.

*Three patients grew two pathogens.

$\mathrm{BAL}$, bronchoalveolar lavage; IPF, idiopathic pulmonary fibrosis; WG, Wegener granulomatosis. 
Table 3 Bronchoalveolar lavage fluid cytokine levels

\begin{tabular}{lllll}
\hline & IL1 $\boldsymbol{\alpha}$ & IL1 $\beta$ & IL1ra & IL6 \\
\hline Normal & Not detected & $3.8(2.2-4.0)$ & $755(165-1212)$ & $0.3(0-0.5)$ \\
WG all patients & $58.9(0-84.9) \dagger$ & $13.21(4.0-41.5) \#$ & $4957(1453-36819) \dagger$ & $2.3(1.0-16.8) \dagger$ \\
WG pathogen present & $28.1(0-77.7)$ & $5.0(4.0-38.5)$ & $5,824(4085-45175)^{*}$ & $3.5(2.1-15.7)$ \\
WG no pathogen & $84.6(38.11)$ & $4.0(4.0-50.6)$ & $3307(1112-11275)$ & $1.8(1.3-16.8)$ \\
IPF all patients & $33.7(0-50.0)$ & $0.5(0.5-23.9)$ & $8061(1450-22104) \dagger$ & $8.4(3.5-17.4) \dagger$ \\
IPF pathogen present & $36.5(0-74.5)$ & $10.3(0.5-36.9)$ & $22,104(2890-33978)^{*}$ & $13.4(6.0-22.1)$ \\
IPF no pathogen & $18.9(0-21.6)$ & $1.0(0.5-5.0)$ & $3008(628-8002)$ & $3.5(2.7-12.9)$ \\
\hline
\end{tabular}

Measurements are expressed at $\mathrm{pg} / \mathrm{ml}$ concentration. Levels are expressed as median (interquartile range).

*Statistically significant difference between pathogen present and pathogen not present. †Statistically significant difference between disease group and normal controls.

IL, interleukin; IL1ra, interleukin1 receptor antagonist; IPF, idiopathic pulmonary fibrosis; WG, Wegener granulomatosis.

with WG compared with normal controls. IL1ra $(p<0.001)$ and IL6 $(p=0.009)$ levels were elevated in patients with IPF compared with controls. IL1 $\alpha(p=0.05)$ and IL1 $\beta(p=0.001)$ levels were higher in patients with WG compared with IPF disease controls. Levels of IL1ra were elevated in patients with WG $(p=0.039)$ and IPF $(p=0.015)$ who grew a pathogen compared with those who did not. The other cytokine levels measured did not relate to the presence or absence of a pathogen (table 3).

\section{Soluble factors within BALF promote $\boldsymbol{S}$ aureus growth}

Incubations with BALF from patients with WG promoted statistically significantly more $S$ aureus growth (Dunn test, 168.4 CFU/ml (IOR 95-268), $p=0.025$ ) than BALF from patients with IPF $[75.0 \mathrm{CFU} / \mathrm{ml}(\mathrm{IOR} 37-122), \mathrm{p}=0.041)$ and normal controls (23 CFU/ml (IOR 19-42)). Heat inactivation resulted in a statistically significant reduction in $S$ aureus growth with BALF from patients with WG (23.5 CFU/ml (IOR 3-63), ( $p=0.0008)$ (fig 1).

\section{DISCUSSION}

This study has shown that pathogens were more commonly isolated from BALF of patients with WG and IPF than that from

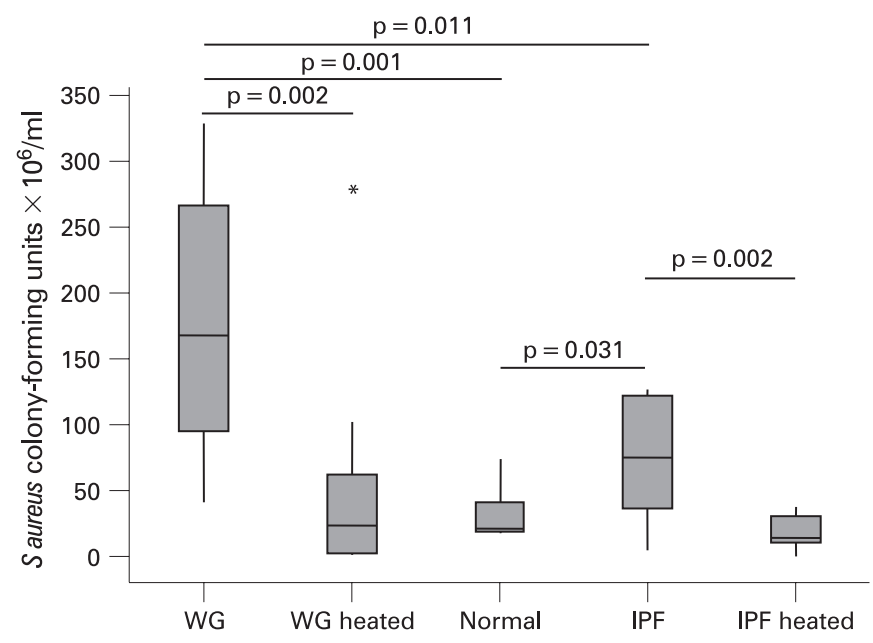

Figure $1 S$ aureus growth in culture with brochoalveolar lavage fluid (BALF). BALF or normal saline (50:50 mix) with brain heart infusion were incubated with an inoculum of $10^{-5}$ bacteria per ml. After $24 \mathrm{~h}$ of incubation, quantitative culture was performed to determine the number of $S$ aureus colony-forming units. BALF experiments were repeated following heat inactivation of the proteins in the BALF for $10 \mathrm{~min}$ at $80^{\circ} \mathrm{C}$. IPF, idiopathic pulmonary fibrosis; WG, Wegener granulomatosis. normal controls. S aureus was most frequently found in the WG remission and relapse groups rather than at disease presentation. Serial bronchoscopy suggests that pathogen presence in BALF was due to transient infection rather than persistent lower airway colonisation. There were no significant associations between pathogen growth and radiological findings, pulmonary function tests, immunosuppression or antibiotic treatment. Culture experiments revealed that WG BALF was a permissive environment for $S$ aureus growth and that this effect was heat sensitive. Also, cytokines, previously reported to be promotive of $S$ aureus growth in vitro, were elevated in WG.

Previous studies looking at nasal carriage of $S$ aureus have suggested that persistent nasal $S$ aureus carriage is associated with disease relapse in WG. ${ }^{2}$ We believe that ours is the first study to demonstrate that lower airway bacterial infection, especially with $S$ aureus, is also common in patients with WG. Chronic lung damage is a recognised feature of the WG lung. ${ }^{14}$ and this may contribute to pathogen growth in conjunction with immunosuppressant therapy. Previous studies have shown that with progressive epithelial cell damage, adherence and internalisation of $S$ aureus are increased. ${ }^{3}$ In WG this may be important since ANCA itself also induces IL1 and IL8 release from neutrophils, ${ }^{15}$ potentially amplifying local inflammatory tissue damage and thereby promoting $S$ aureus growth.

The mechanisms by which $S$ aureus may have a predilection for the lower airways of patients with WG especially in chronic disease are likely to be multifactorial-related to structural changes, functional immunodeficiency, defective host innate immunity or variability in the virulence of $S$ aureus strains. There was a high incidence of bronchiectasis and cavities in the patients with WG relapse and remission, but this did not relate to the presence of a pathogen. Equally most but not all patients who had lower airway $S$ aureus growth also had $S$ aureus in the nose, suggesting a link with nasal carriage as part of the general respiratory tract response.

The lack of association with pulmonary features investigated in this study may reflect the relatively low numbers recruited in this trial, due to rarity of WG rather than a true lack of effect. However, if the lack of relationship is true it is likely that, in contrast to cavitating staphylococcal pneumonia, ${ }^{16}$ the underlying disease process rather than infection is driving the cavitation process, especially since several cases of cavitating disease had completely sterile BALF. There was also no clear effect of immunosuppression or chemoprophylaxis on bacterial growth, although the data were difficult to interpret because of the relatively small numbers in each group. Bacteria were isolated in active and remission patients despite the prophylactic co-trimoxazole prescribed, demonstrating that this dose was inadequate to control pathogen growth. 
The microbiology pattern in WG was different between our three defined activity groups, with $S$. aureus more commonly isolated in disease relapse and remission patients rather than at acute presentation, suggesting that $S$ aureus may have a role in disease relapse. The predilection of WG for $S$ aureus infection appears to be disease related as these changes were not seen in patients with IPF who we used as a pulmonary disease control. Due to the intense inflammatory response associated with WG activity, we were unable to associate the presence of a pathogen with the intensity of the local cellular inflammatory response to confirm that this represented an infection rather than colonisation. However, BALF IL1Ra levels were increased in association with the presence of a pathogen in patients with both WG and IPF.

IL1Ra is an acute phase proteins and staphylococci are highly efficient in mediating the upregulation of IL $1 \alpha$, IL1 $\beta$ and IL1Ra. ${ }^{17}$ These data suggest that the presence of staphylococci may induce or relate to a local alveolar inflammation. In addition, since IL1Ra has previously been shown to be a trophic factor for $S$ aureus, ${ }^{4}$ we performed relevant but simple BALF growth experiments in vitro. The observation that BALF from patients with WG stimulated growth of $S$ aureus supports a role for a trophic interaction between the host inflammatory response and the bacteria within the lung. In this regard, WG BALF appears to have similar properties to that of acute respiratory distress syndrome. ${ }^{4}$

There are a number of potential mechanisms whereby $S$ aureus infection might initiate relapse in WG; $S$ aureus is able to stimulate directly the tumour necrosis factor $\alpha$ receptor, ${ }^{17}$ superantigen release, ${ }^{18}$ generation of immune complexes, ${ }^{19}$ expansion of memory effector $\mathrm{T}$ cells ${ }^{20}$ and may induce glucocorticoid resistance ${ }^{21}$ Further recruitment, in a longitudinally studied cohort, will be required to assess the importance of our findings, and to address whether the prevalence of $S$ aureus in the lower airway is associated with an increased risk of subsequent disease relapse, thus making intervention relevant.

This study has also demonstrated that a significant number of patients with IPF grow pathogenic bacteria from their BALF with a spectrum of pathogens different from WG. We specifically excluded any patient thought clinically to have had evidence of infection within the 4 weeks prior to bronchoscopy, and on their first bronchoscopy they were not immunosuppressed. To our knowledge, no study has previously looked at the issue of bacterial colonisation in IPF. Given the variable presence of cellular neutrophilic inflammation in patients with IPF, it is possible therefore that unrecognised airway colonisation is a determinant of this phenomenon. The clinical relevance of this observation warrants further evaluation especially in view of the recent publication of a pilot study demonstrating improved exercise tolerance and lung function with continuous co-trimoxazole usage in IPF patients. ${ }^{22}$

This study has limitations. First, during intubation, oralpharyngeal contamination of the bronchoscope could have contributed to BALF pathogen growth. To counter this potential problem we used quantitative culture, a microbiological culture technique that has evolved as a sensitive and specific technique for the diagnosis of bacterial infection. ${ }^{23}$ Serial dilutions help to overcome contamination of the bronchoscope suction channel by oropharyngeal bacteria, ${ }^{24}$ and results were greater than would be expected from contamination alone. Secondly, we are unable to demonstrate in our BALF growth experiments that IL1Ra is directly responsible for the growthpromoting potential within the BALF as there is no effective inhibitor of IL1ra bioactivity. Finally, this was an observational study to ascertain the prevalence of pathogenic bacteria in the lungs of patients with WG, and our results need validation in another cohort of patients with WG.

In summary, this study has found a high incidence of pathogen carriage in the lower airways of patients with WG and IPF. The predilection for $S$ aureus in WG is likely to be multifactorial but may relate to a dynamic relationship between alveolar inflammation and bacterial growth. Determining the role and mechanisms of lung $S$ aureus carriage in disease and relapse may provide strategies for future treatments in WG. Physicians treating patients with acute or relapsing WG who wish to treat potential pulmonary infection should consider bronchoscopic microbiological sampling and prescribing antibiotics with antistaphylococcal activity.

Acknowledgements: We would like to thank the staff and patients of the Birmingham vasculitis clinic and the University Hospital ILD clinic. We would like to thank Professor Caroline Savage and Dr Peter Guest (PG) for their help in this project, and Patti Bharadwa for her technical assistance.

Funding: AR was supported by a Wellcome entry level fellowship. DRT is supported by a Wellcome Intermediate Fellowship.

\section{Competing interests: None.}

Ethics approval: This study was approved by the local ethics committee (South Birmingham, UK, LREC ref 2003/166).

\section{REFERENCES}

1. Pinching AJ, Rees AJ, Pussell BA, et al. Relapses in Wegener's granulomatosis: the role of infection. Br Med J 1980;281:836-8.

2. Stegeman CA, Tervaert JW, Sluiter WJ, et al. Association of chronic nasal carriage of Staphylococcus aureus and higher relapse rates in Wegener granulomatosis. Ann Intern Med 1994;120:12-7.

3. Kanangat S, Bronze MS, Meduri GU, et al. Enhanced extracellular growth of Staphylococcus aureus in the presence of selected linear peptide fragments of human interleukin (IL)-1 beta and IL-1 receptor antagonist. J Infect Dis 2001;183:65-9.

4. Meduri GU, Kanangat S, Stefan J, et al. Cytokines IL-1beta, IL-6, and TNF-alpha enhance in vitro growth of bacteria. Am J Respir Crit Care Med 1999;160:961-7.

5. Tang YW, Johnson JE, Browning PJ, et al. Herpesvirus DNA is consistently detected in lungs of patients with idiopathic pulmonary fibrosis. J Clin Microbiol 2003;41:2633-40.

6. Strieter RM. Pathogenesis and natural history of usual interstitial pneumonia: the whole story or the last chapter of a long novel. Chest 2005;128(5 Suppl 1):526S-32S.

7. Stewart JP, Egan JJ, Ross AJ, et al. The detection of Epstein-Barr virus DNA in lung tissue from patients with idiopathic pulmonary fibrosis. Am J Respir Crit Care Med 1999;159:1336-41.

8. Jennette JC, Falk RJ, Andrassy K, et al. Nomenclature of systemic vasculitides. Proposal of an international consensus conference. Arthritis Rheum 1994;37:187-92.

9. Merkel PA, Cuthbertson DD, Hellmich B, et al. Comparison of disease activity measures for anti-neutrophil cytoplasmic autoantibody (ANCA)-associated vasculitis. Ann Rheum Dis 2009;68:103-6.

10. Luqmani RA, Bacon PA, Moots RJ, et al. Birmingham Vasculitis Activity Score (BVAS) in systemic necrotizing vasculitis. OJM 1994;87:671-8.

11. American Thoracic Society. Idiopathic pulmonary fibrosis: diagnosis and treatment. International consensus statement. American Thoracic Society (ATS), and the European Respiratory Society (ERS). Am J Respir Crit Care Med 2000;161:646-64.

12. Cordier JF, Valeyre D, Guillevin L, et al. Pulmonary Wegener's granulomatosis. A clinical and imaging study of 77 cases. Chest 1990;97:906-12.

13. British Thoracic Society Bronchoscopy Guidelines Committee, a Subcommittee of Standards of Care Committee of British Thoracic Society. British Thoracic Society guidelines on diagnostic flexible bronchoscopy. Thorax 2001;56(Suppl 1):i1-21.

14. Newall C, Schinke S, Savage CO, et al. Impairment of lung function, health status and functional capacity in patients with ANCA-associated vasculitis. Rheumatology (Oxford) 2005;44:623-8.

15. Harper L, Ren Y, Savill J, et al. Antineutrophil cytoplasmic antibodies induce reactive oxygen-dependent dysregulation of primed neutrophil apoptosis and clearance by macrophages. Am J Pathol 2000;157:211-20.

16. dos Santos JW, Nascimento DZ, Guerra VA, et al. Community-acquired staphylococcal pneumonia. J Bras Pneumol 2008;34:683-9.

17. Megyeri K, Mandi Y, Degre M, et al. Induction of cytokine production by different Staphylococcal strains. Cytokine 2002;19:206-12.

18. Popa ER, Stegeman CA, Bos NA, et al. Staphylococcal superantigens and T cell expansions in Wegener's granulomatosis. Clin Exp Immunol 2003;132:496-504.

19. Brons RH, Bakker HI, Van Wijk RT, et al. Staphylococcal acid phosphatase binds to endothelial cells via charge interaction; a pathogenic role in Wegener's granulomatosis? Clin Exp Immunol 2000;119:566-73. 
20. Abdulahad WH, Stegeman CA, Limburg PC, et al. CD4-positive effector memory T cells participate in disease expression in ANCA-associated vasculitis. Ann NY Acad Sci 2007;1107:22-31

21. Li LB, Goleva E, Hall CF, et al. Superantigen-induced corticosteroid resistance of human $T$ cells occurs through activation of the mitogen-activated protein kinase kinase/extracellular signal-regulated kinase (MEK-ERK) pathway. J Allergy Clin Immunol 2004;114:1059-69.
22. Varney VA, Parnell HM, Salisbury DT, et al. A double blind randomised placebo controlled pilot study of oral co-trimoxazole in advanced fibrotic lung disease. Pulm Pharmacol Ther 2008;21:178-87.

23. Kahn FW, Jones JM. Diagnosing bacterial respiratory infection by bronchoalveolar lavage. J Infect Dis 1987;155:862-9.

24. Meduri GU, Beals DH, Maijub AG, et al. Protected bronchoalveolar lavage. A new bronchoscopic technique to retrieve uncontaminated distal airway secretions. Am Rev Respir Dis 1991;143:855-64.

\section{Pulmonary puzzle}

\section{ANSWER}

From the question on page 682

Considering the remarkable response to chemotherapy, the new lung nodule did not seem to be a metastatic lesion of the primary lung cancer. Percutaneous needle aspiration showed abundant aspergillus fungal hyphae on specimens. Taking the clinical course into account, a final diagnosis of chronic necrotising pulmonary aspergillosis was made (fig 1A). After treatment with voriconazole for 6 months, only the fibrotic scar remained and the medication was discontinued (fig 1B). Complete response of the lung cancer was obtained with further chemoradiotherapy and has been sustained for almost 2 years to date.

Aspergillus is a ubiquitous saprophytic fungus which causes a variety of human illnesses ranging from allergic reactions to aggressive forms invading the lung, brain and other organs. ${ }^{1}$ Invasive aspergillosis has become an important infectious complication in patients with cancer, with a high fatality rate as a result of severe neutropenia. ${ }^{2}$ It is also noteworthy that lung cancer seems to be more frequently associated with secondary aspergillosis than was previously thought. ${ }^{3}$ In the early 1980s Geftner et al and Binder et al described a new category of lung disease caused by aspergillus in patients with underlying structural lung disease or mild immunosuppression including diabetes mellitus, alcoholism, chronic liver disease and corticosteroid use. ${ }^{45}$ In our case, altered host immunity caused by chemotherapy might have triggered colonisation by the fungus on the fibrotic area to grow into the lung parenchyma. To our knowledge, this is the first case of chronic necrotising pulmonary aspergillosis developing during chemotherapy for a solid tumour.

Thorax 2009;64:697. doi:10.1136/thx.2008.106260a

\section{REFERENCES}

1. Soubani A0, Chandrasekar PH. The clinical spectrum of pulmonary aspergillosis. Chest 2002;121:1988-99.

2. Maschmeyer G, Haas A. The epidemiology and treatment of infections in cancer patients. Int J Antimicrob Agents 2008;31:193-7.

3. Shahid M, Malik A, Bhargava R. Bronchogenic carcinoma and secondary aspergillosis. Cancer 2008:113:547-58.

4. Gefter WB, Weingrad TR, Epstein DM, et al. "Semi-invasive" pulmonary aspergillosis: a new look at the spectrum of aspergillus infections of the lung. Radiology 1981:140:313-21.

5. Binder RE, Faling LJ, Pugatch RD, et al. Chronic necrotizing pulmonary aspergillosis: a discrete clinical entity. Medicine (Baltimore) 1982:61:109-24.
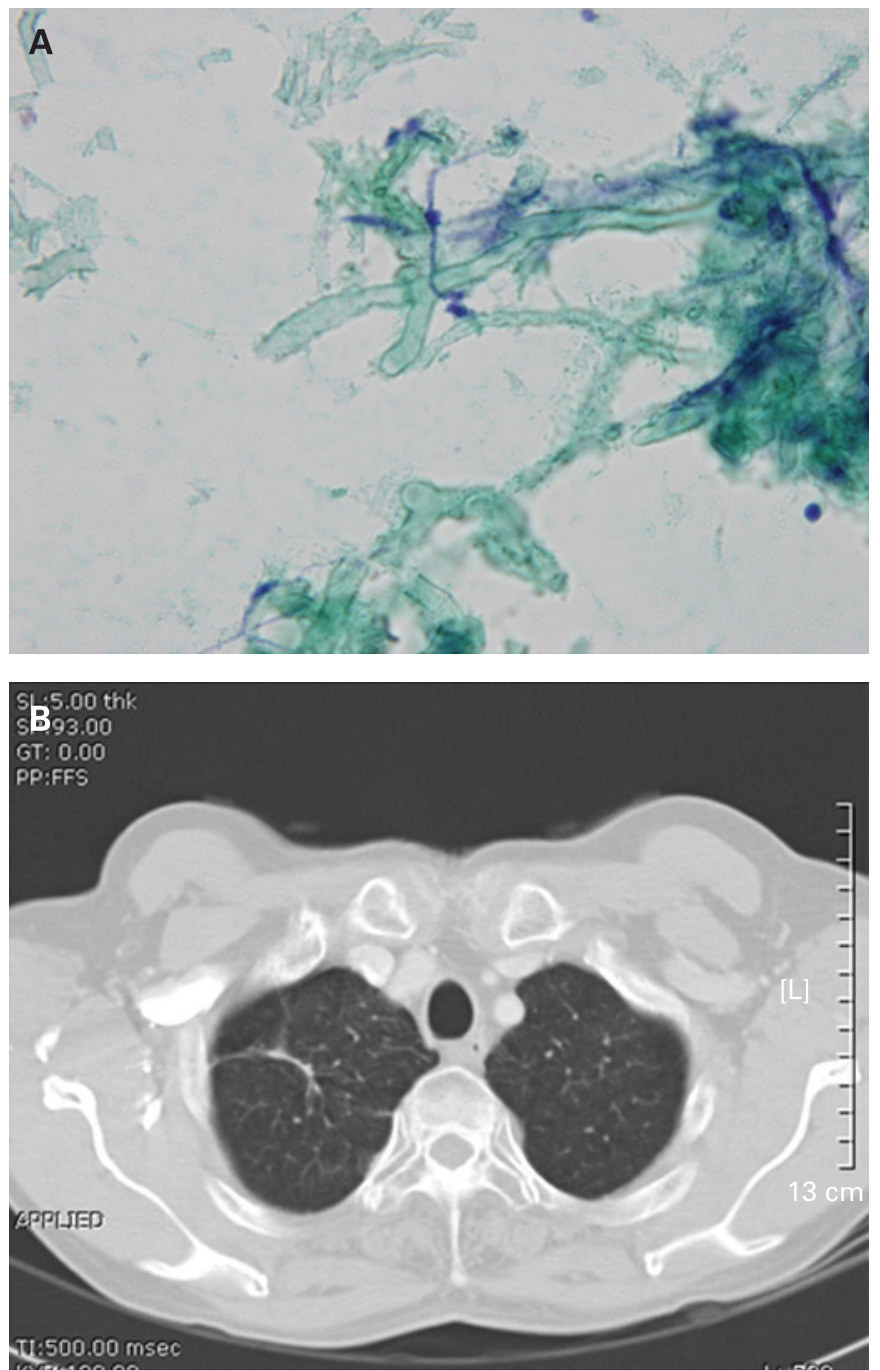

Figure 1 (A) Histological examination of aspirated specimens showing aspergillus fungal hyphae (Papanicolaou stain, $\times 400$ ). (B) CT scan obtained 6 months after antifungal treatment showing complete resolution of the nodule with residual parenchymal scar formation. 